\title{
Nutrition training in UK medical undergraduate programmes
}

\author{
R. D. Johnston ${ }^{1,3}$, P. Neild ${ }^{2}$, Y. M. Jeanes ${ }^{3}$ and T. E. Bowling ${ }^{1}$ \\ ${ }^{1}$ Wolfson Digestive Diseases, Nottingham NG7 2UH, UK, ${ }^{2}$ St George's Hospital, London, SW17 ORE, UK \\ and ${ }^{3}$ Roehampton University, London SW15 5PU, UK
}

An increasing number of UK patients suffer from nutrition-related illnesses. Training in nutrition at medical schools has previously been shown to be inadequate ${ }^{(1)}$. Educational programmes designed to increase the quantity of nutrition teaching have been shown to improve student knowledge and satisfaction ${ }^{(2)}$.

The aim of the present study was to survey the nutrition training provided to medical undergraduates in the UK. A thirteen-item questionnaire was sent to the deans and lead nutrition clinicians of all UK medical schools. Teaching in nutrition was defined specifically as that covering the interaction between diet and health, as opposed to merely basic physiology or biochemistry. The data generated was analysed using SPSS version 15.0 (SPSS Inc., Chicago, IL, USA) and significance was determined using a two-tailed independent samples $t$ test.

The response rate was $63 \%$ (twenty of thirty-two). The respondents were members of the deanery $(n 7)$, lecturers or educationalists ( $n$ 5) and clinicians responsible for teaching nutrition ( $n$ 7); data was absent from one respondent. The courses were described as being traditional ( $n$ 5), integrated ( $n$ 7), problem-based $(n 6)$ and graduate entry $(n 2)$.

Nutrition was reported as having a high priority in the planning of the curriculum by one respondent, intermediate by twelve and low by seven. Student's knowledge of nutrition was assessed in all but two medical schools. The mean period (h) of structured teaching in nutrition throughout the courses was 19 (range 2-50), although respondents reported difficulty in giving a precise answer to this question. Eleven schools $(55 \%)$ responded that they taught all the following core areas as part of their undergraduate curriculum: the nutritional assessment of patients; health consequences of malnutrition; practicalities of nutritional support; dietary treatments of specific disease states. Four of the twenty schools reported that they did not teach the health consequences of malnutrition.

Ten of twenty respondents $(50 \%)$ reported that their students were not adequately trained in nutrition in order to become effective doctors, whereas only six stated that their students wished for greater teaching.

Courses that reported a high or intermediate priority for nutrition in their curriculum were associated with a greater mean period (h) allocated (29 v. 9; $P=0.01$ ), greater likelihood of teaching all four core areas (ten of thirteen $v$. one of seven; $P<0.01$ ), reporting of adequate training (seven of twelve $v$. one of six; $P=0.086$ ) and contented student feedback (five of eleven $v$. one of six; $P=0.016$ ).

In conclusion, student training and satisfaction appears to be greater in courses where nutrition was given a higher priority. Half the respondents felt that their courses inadequately trained students in nutrition for them to become effective doctors. The amount of time allocated to teaching nutrition appears to be low and the coverage of core areas was incomplete in $45 \%$ of courses.

The incomplete response rate to this questionnaire may reflect the low priority for nutrition among some medical schools. The true picture of education in nutrition in the UK may therefore be worse than that presented here. In order to have adequately-trained doctors it is imperative to increase the amount, depth and priority of nutrition in UK medical school curricula and assessment processes.

1. Adams KM, Lindell KC, Kohlmeier M et al. (2006) Am J Clin Nutr 83, 941S-944S.

2. Taren DL, Thomson CA, Koff NA et al. (2001) Am J Clin Nutr 73, 1107-1112. 